\title{
Mise au point Estrogènes et reproduction chez le mâle
}

\author{
S. CARREAU
}

Laboratoire Biochimie-IRBA, UPRES EA 2608, Université, 14032 CAEN

\section{RESUME}

L'aromatase est l'enzyme terminale qui catalyse irréversiblement la transformation des androgènes en estrogènes. Outre les cellules somatiques du testicule, la mise en évidence d'une source supplémentaire d'estrogènes dans les cellules germinales, associée à la présence de récepteurs spécifiques (ER $\alpha$ et ERß), plaide fortement en faveur d'un rôle physiologique pour ces hormones femelles dans la régulation des fonctions testiculaires chez certains mammifères. De fait chez la souris et chez l'homme, une déficience en aromatase est mise en cause en particulier dans les anomalies osseuses souvent associées à une stérilité. En conséquence, la régulation des fonctions testiculaires fait non seulement intervenir les gonadotrophines et les androgènes mais l'estradiol (ou le rapport androgène / estrogène) semble de plus en plus concerné, en agissant soit directement soit par l'intermédiaire des cellules somatiques, dans la production et la maturation du spermatozoïde.

Mots clés: Estrogènes, spermatogenèse, récepteurs estrogènes, aromatase, fertilité, homme
AVANT PROPOS

Le testicule de mammifêres est caractérisé par deux fonctions majeures: l'une exocrine, la production des gamètes mâles et l'autre endocrine, la synthèse des stéroïdes ; ces deux fonctions sont sous le contrôle des gonadotrophines, dont l'action est modulée in situ par de nombreuses substances produites par les cellules gonadiques elles-mêmes (pour revue [39]). Les estrogènes, longtemps considérés comme des hormones sexuelles typiquement femelles, font l'objet aujourd'hui de nombreux travaux et leur présence dans le testicule est très documentée (pour revue [6]). Depuis longtemps on sait que la secrétion des gonadotrophines est contrôlée négativement par les hormones femelles; cependant des évidences nombreuses plaident en faveur d'un rôle physiologique direct des estrogènes au niveau testiculaire et épididymaire.

\section{L'AROMATASE ET LES SOURCES D'ESTROGENES DANS LE TESTICULE}

L'aromatase est l'enzyme qui catalyse de manière irréversible la transformation des androgènes en estrogènes. Elle est impliquée notamment dans le développement, la reproduction, la différenciation sexuelle et le comportement, les métabolismes osseux et lipi-

Correspondance: Dr S.CARREAU Tél: 0231565488 Fax : 0231565320 E-mail: carreau@ibba.unicaen.fr 
dique, le fonctionnement du cerveau; son importance est donc capitale chez l'homme [5].

Cette enzyme est une glycoprotéine membranaire composée de deux entités : la cytochrome P450 aromatase (P450arom), une glycoprotéine héminique spécifique, et une réductase ubiquiste. Chez les mammifères, dans le réticulum endoplasmique de pratiquement tous les tissus (en particulier le cerveau, les gonades, le placenta, la glande mammaire et les tissus adipeux et osseux) on trouve ce complexe enzymatique. Le gène de l'aromatase humaine (CYP 19 ) est composé de 18 exons dont 9 codants, il est localisé dans la région q21.1 du chromosome 15 (pour revues [46,47]). Dans la partie 5' non-codante du gène sont situés plusieurs promoteurs avec des éléments régulateurs spécifiques ce qui fait que l'expression de CYP19 dépend de l'environnement cellulaire. Dans la gonade mâle, le promoteur PII est activé préférentiellement mais un second promoteur appelé I.6 (similaire au promoteur I.3 du tissu adipeux, en termes de régulation et d'expression) existe et son expression est plus forte dans le tissu tumoral [44].

Dans le tissu testiculaire des mammifères, on sait depuis plusieurs décennies que l'aromatase est essentiellement localisée dans les cellules de Leydig; mais récemment chez les rongeurs, cette protéine a été aussi mise en évidence dans les cellules germinales [6]. L'activité aromatase est effectivement présente non seulement dans les cellules de Leydig mais aussi dans les cellules de Sertoli et, fait nouveau, dans les cellules germinales: l'activité enzymatique est plus forte dans les spermatozoïdes que dans les spermatocytes pachytènes [29]. En utilisant des sondes moléculaires spécifiques, la présence d'ARN messager de l'aromatase a été démontrée dans les cellules de Leydig et de Sertoli ainsi que dans les cellules germinales (spermatocytes, spermatides et à un degré moindre spermatozoïdes). L'existence de cette protéine biologiquement active dans les cellules germinales haploïdes, notamment les spermatides allongées et les spermatozoïdes, est corroborée par les travaux de Janulis et al. [26, 27]; ces auteurs ont montré que la protéine et l'activité enzymatique décroissent au cours du transit épididymaire chez le rat et la souris, suggérant ainsi que l'aromatase serait localisée au niveau de la gouttelette cytoplasmique du spermatozoïde. Nos résultats [3, 29] et ceux de la littérature apportent la preuve que les cellules sexuelles représentent une source supplémentaire d'estrogènes dans le testicule, notamment chez les rongeurs et les ursidés $[7,8]$.

Ces données nouvelles relatives aux estrogènes testiculaires ont été confortées par les travaux de l'équipe de Simpson [16,37] : ces auteurs ont démontré que des souris mâles déficientes en aromatase ou ArKO (suite à l'insertion d'un néogène entre deux sites de restriction de l'exon $\mathrm{X}$ ) sont d'abord fertiles puis une infertilité progressive apparaît chez les animaux dès l' âge de 4 mois. Cette anomalie qui concerne tous les animaux âgés d'un an, est marquée par un arrêt de maturation des spermatides associé à une diminution de leur nombre. Outre des anomalies au niveau de l'acrosome, une augmentation de l'apoptose a été observée dans le compartiment adluminal : les spermatides sont multinucléées et dégénérescentes.Chez les mâles homozygotes pour la mutation, les taux de LH et testostérone augmentent alors que celui de la FSH reste normal. En conséquence, les animaux présentent une hyperplasie / hypertrophie leydigienne due à l'élévation des taux de LH; cependant le volume des tubes séminifêres reste inchangé ainsi que les nombres de cellules de Sertoli, spermatogonies et spermatocytes. En somme ces données suggèrent donc chez les souris ArKO la présence d'une anomalie spécifique centrée sur les spermatides (Figure 1).

Pourquoi les animaux sont-ils fertiles puis deviennent-ils infertiles? une alimentation riche en soja (donc en phytoestrogènes) a été évoquée, mais les derniers travaux du groupe australien écartent cette éventualité [38]. Une autre hypothèse actuellement à l'étude fait appel à d'autres ligands (androgéniques: $5 \alpha$ androstanediol; facteurs de croissance: type EGF) capables d'activer le récepteur aux estrogènes dans les cellules germinales (voir paragraphe suivant). A l'opposé du modèle ArKO, des souris transgéniques qui surexpriment l'aromatase sont infertiles et des tumeurs leydigiennes apparaissent [18]. 

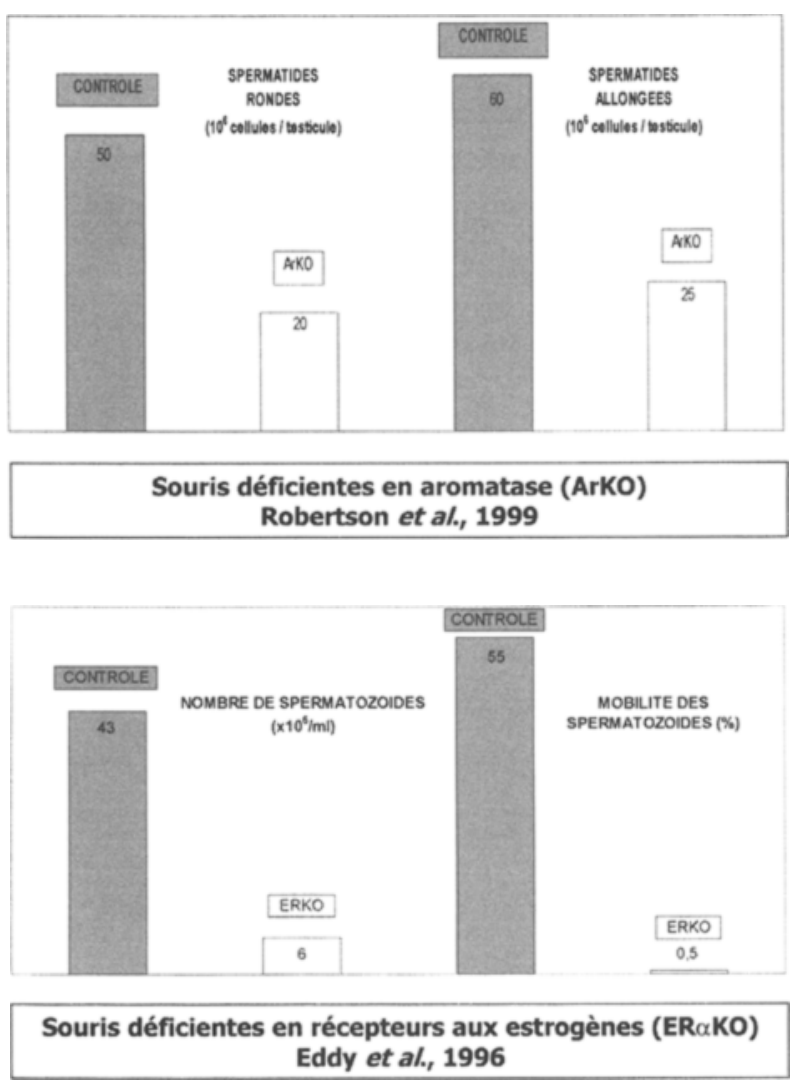

Figure 1: Spermatogenèse et caractéristiques des spermatozoïdes chez les souris déficientes en aromatase (ArKO) ou en récepteurs aux estrogènes $(\boldsymbol{E R} \alpha \boldsymbol{K} \boldsymbol{O})$.

\section{Pathologie humaine}

La source d'estrogène testiculaire chez l'homme est représentée par les cellules de Leydig $[25,34]$ bien qu'in vitro les cellules de Sertoli puissent produire de l'estradiol (pour revue [7]). Plus récemment la capacité du spermatozoïde humain a métaboliser la pregnénolone en dérivés delta 4-3 cétoniques , dont la testostérone et l'estradiol, a été décrite [19]; de fait il semblerait que les spermatozoïdes expriment l'aromatase puiqu'un ARNm spécifique vient d'être détecté par notre équipe (Lambard, Denis-Galeraud et Carreau, non publiés).

Chez l'homme quelques cas de déficit en aromatase par mutation sur le gène CYP 19 ont été publiés : la pathologie se traduit par des troubles de la croissance (homme de très grande taille), un hyperinsulinisme et une perturbation du bilan lipidique. Les concentrations en gonadotrophines sont très élevées alors que celles de l'estrone et de l'estradiol sont indétectables (confirmant le contrôle négatif exercé par les estrogènes sur la secrétion des gonadotrophines) mais aucun spermogramme n'a été fait (pour revue [15]). Les observations sont donc peu documentées quant à l'impact du déficit en aromatase sur la fertilité des patients, seuls Carani et al [4] ont publié un tableau d'infertilité masculine chez un patient présentant une mutation homozygote inactivant le gène de l'aromatase : hypotrophie testiculaire associée à une oligo-asthénospermie très sévère ( 1 million de spermatozoïdes / $\mathrm{ml}$ à $100 \%$ immobiles). Ce patient traité par l'estradiol a vu ses paramètres hormonaux revenir dans la normale alors que la testostérone était inefficace. Contrairement au patient de Morishima et al [32] qui présentait une macroorchidie, celui de Carani et al [4] avait des testicules petits. Quel que soit le patient, la déficience en aromatase porte toujours sur des mutations d'acides aminés situés dans la région protéique concernée dans l'activité enzymatique (pour revue [20]). Toutefois il convient de souligner qu'aucune corrélation entre l'absence d'aromatase et l'azoospermie (infertilité) n'a pu être mise en évidence puisque l'un des frères du patient présentait la même pathologie testiculaire avec un P450arom normal [15] ce qui doit conduire à de nouvelles études dans ce domaine pour préciser l'éventuel lien de causalité entre déficience en estrogènes et infertilité chez l'homme [15, 48].

\section{ESTROGENES ET FONCTIONS DE REPRODUCTION}

Comme beaucoup de ligands stéroïdiens, les estrogènes agissent par l'intermédiaire de récepteurs spécifiques (ER) qui en tant que facteur de transcription vont moduler l'expression de gènes cibles. Avec la découverte en 1996 d'un second récepteur nucléaire dénommé ERß $[21,33]$, le rôle des estrogènes, notamment au niveau testiculaire, fait l'objet de nombreuses recherches (pour revues $[6,14,41]$ ). En effet, ERß est présent dans les cellules de Sertoli et les germinales (spermatides rondes et à un degré moindre dans les spermatocytes pachytènes) alors que $\mathrm{ER} \alpha$ est principalement localisé dans les cellules de Leydig chez le rat [45, 51] (Tableau 1). 
Tableau 1. Récepteurs aux estrogènes (ER $\alpha-E R \beta)$ et activité aromatase dans les cellules testiculaires et le tractus génital du rat mâle adulte.

\begin{tabular}{lccc}
\hline Tissu /Cellule & ER $\alpha$ & ERß & Aromatase \\
\cline { 4 - 5 } Cellule de Leydig & + & $+/-$ & + \\
Cellule péritubulaire & - & - & $+/-$ \\
Cellule de Sertoli & - & + & + \\
Spermatogonie & - & + & NR \\
Spermatocytes & & & \\
pachytène & - & + & + \\
Spermatides rondes & - & + & + \\
Spermatozoïde & $+?$ & + & + \\
Rete testis & + & $\mathbf{N R}$ & $\mathbf{N R}$ \\
Canaux efferents & + & + +- & NR \\
Epididyme & $+/-$ & + & $\mathbf{+ ?}$ \\
Vésicules séminales & - & + & $\mathbf{N R}$ \\
Prostate & - & + & $\mathbf{+ ?}$ \\
\hline
\end{tabular}

NR: non recherché

Chez l'homme, Enmark et al [13] ont montré que les spermatides rondes en particulier exprimaient ERß, un signal plus faible est détectable dans les spermatocytes pachytènes alors que les cellules somatiques sont négatives. Par ailleurs il existerait deux isoformes de ERß : ERß1 est surtout présent dans les spermatides, à un degré moindre dans les Sertoli, spermatogonies et spermatocytes pachytènes. Par contre, ERß2 (qui ne lie pas le ligand estrogénique) est essentiellement localisé dans les Sertoli et les spermatogonies mais absent des spermatocytes et spermatides [40].Ces données mettent clairement en évidence un rôle potentiel des estrogènes au niveau des cellules haploïdes humaines.

Dans les spermatozoïdes humains, un récepteur (identifié par son ARNm et la protéine) aux estrogènes a été mis en évidence par Durkee et $a l$ [11]. A côté de ces récepteurs classiques qui induisent des effets génomiques, l'existence de récepteurs membranaires fonctionnels pour les estrogènes a été rapportée par Luconi et al [30]; cet effet membranaire rapide des estrogènes est plausible compte tenu notamment de l'environnement hormonal dans lequel ces cellules vont baigner dans le tractus génital femelle (pour revue [36]).

Les estrogènes quelle que soit leur origine cellulaire régulent de manière autocrine et / ou paracrine, non seulement la stéroïdogenèse mais très probablement aussi la spermatogenèse. Citons par exemple la prolifération des gonocytes et la synthèse des cadhérines qui sont dépendantes des estrogènes [6]. En ce qui concerne le développement des cellules germinales, de nombreux travaux plaident en faveur d'un rôle positif de l'estradiol en particulier chez les mammifères hibernants [7]. Très récemment nous avons pu montrer chez le campagnol qu'il y a synchronisation entre l'expression de l'aromatase et de ERB dans les tubes séminifères (plus précisement dans les spermatides) avec la reprise de la spermatogenèse [2].

Shetty et al. [42, 43] ont observé qu'un inhibiteur de l'aromatase (dérivé du letrozole) injecté chez le singe induisait une interruption de la maturation et du nombre des cellules germinales (diminution de $90 \%$ du nombre des spermatides associée à une chute de la motilité des spermatozoïdes) ce qui indéniablement plaide en faveur du rôle positif des estrogènes dans la spermiogenèse des primates; ces observations sont d'ailleurs en accord avec les données observées chez les souris ArKO [37]. Cependant des travaux récents portant sur le traitement du rat mâle avec un autre inhibiteur de l'aromatase, l'anastrozole, n'ont pas permis de mettre en évidence une altération significative de la spermatogenèse bien que certains tubes séminifêres soient dépourvus de cellules germinales [50].

L'invalidation du gène codant soit pour ER $\alpha$ soit pour ERß a permis d'obtenir des animaux de phénotypes différents entre eux (pour revue[9]) et de celui observé chez les ArKO. Chez les souris ER $\alpha \mathrm{KO}$ homozygotes après un développement gonadique pré-et post-natal normal, les poids testiculaires diminuent et une infertilité se développe (la motilité des spermatozoïdes est altérée) (Figure 1); par ailleurs il convient d'ajouter que l'ensemble du tractus génital est affecté [24]. En fait, suite à une dilatation des tubes séminifêres et des canaux efférents, due à une insuffisance de la 
réabsorption du fluide au niveau de l'épididyme, ce dernier reflue dans le tube séminifêre entraînant par augmentation de pression, une dilatation et une destruction mécanique des cellules avoisinantes [23]. Ces observations sont à corréler avec les travaux de Fisher et al [17] qui ont montré que les estrogènes modulent l'expression d'une protéine canal impliquée dans la réabsorption du fluide, l'aquaporine 1, dans les cellules épithéliales des canaux efférents et de la région proximale épididymaire chez le rat et le singe.

Quant aux souris ERßKO (âgées de 6 à 12 semaines), aucune anomalie de la spermatogenèse n'a été jusqu'à présent constatée ; toutefois chez les animaux âgés, l'équipe de Gustafsson signale que les mâles développent une hyperplasie des épithéliums au niveau vésical, séminal et prostatique. Les souris ERKO $\alpha$ et $\beta$, présentent le même phénotype que les souris ER $\alpha K O$, et les mâles sont stériles : le nombre et la mobilité des spermatozoïdes sont réduits respectivement de 80 et $5 \%$ [10]. Les récents résultats publiés par l'équipe de Korach [31] concernant la transplantation de cellules germinales issues des souris mâles $\mathrm{ERKO} \alpha$ chez des mâles receveurs ont clairement prouvé que i) $\mathrm{ER} \alpha$ ne serait pas nécessaire pour le développement de la lignée germinale et ii) l'infertilité des mâles homozygotes ER $\alpha-/-$ serait due au dysfonctionnement des cellules somatiques du testicule.

\section{Pathologie humaine}

En ce qui concerne les récepteurs aux estrogènes une seule pathologie est décrite chez l'homme: il s'agit d'une mutation portant sur le gène où l'exon 2 présente un codon stop en 157 [49]. L'étude du spermogramme a révélé une numération en spermatozoïdes correcte associée à une viabilité réduite $(18 \%)$ avec des volumes testiculaires normaux. Tous ces faits sont à rapprocher de travaux plus anciens rapportant un déficit stéroïdogène et notamment en estrogènes dans le plasma séminal d'hommes azoospermes [6].

\section{CONCLUSION}

En définitive, les cellules germinales du testicule sont capables de synthétiser des estro- gènes. Bien que le rôle exact de ces hormones dans la gonade mâle ainsi que la régulation de l'expression du gène de l'aromatase restent à approfondir, notamment lors du développement testiculaire, ces données associées à celles relatives à la présence de deux types de récepteurs aux estrogènes, suggérent fortement que la physiologie testiculaire est non seulement sous la dépendance des gonadotrophines et de la testostérone mais est aussi contrôlée par l'estradiol. Nous ne sommes finalement qu'au début de cette nouvelle "base de données" où le rôle intracrine des estrogènes est hautement probable compte tenu de l'expression particulière de l'aromatase dans les cellules de la gonade mâle, en particulier chez l'homme (pour revue [22]). Les questions actuelles concernent les gènes estrogénocontrôlés dans les cellules testiculaires; certains d'entre eux codant pour des protéines semblent doivent retenir notre attention: les connexines, en particulier la $\mathrm{Cx} 43$ [1] - le GCNF (Germ cell nuclear factor, un récepteur orphelin apparenté à SF-1) exprimé dans les cellules postméiotiques [28] et potentiellement les cyclines impliquées dans la méiose [35].

\section{Remerciements}

Je voudrais chaleureusement remercier mes collaborateurs (J.Levallet, $C$ Genissel, $S$ Bourguiba, S Lambard, I Denis-Galeraud et B.Bilinska) qui ont contribué à ce travail.

\section{REFERENCES}

1. BATIAS C., DEFAMIE N., LABLACK N., FENICHEL P., SEGRETAIN D., POINTIS G. : Modified expression of testicular gap junction connexin 43 during spermatogenic cycle and in altered spermatogenesis. cell Tissue Res., 1999, 298 : 113-121.

2. BILINSKA B. , SCHMALZ-FRACZEK B., SADOWSKA J., CARREAU S. : Localisation of cytochrome P450 aromatase and oestrogen receptors $\alpha$ and $\beta$ in bank vole testicular cells . Acta Histochem., 2000, 102: 1-15.

3. BOURGUIBA S. , GENISSEL C., BENAHMED M., CARREAU S. : L'aromatase dans les cellules germinales du rat: effets du TGF $\beta$ et du TNF $\alpha$ Andrologie , 2000, sous presse.

4. CARANI C., QIN K., SIMONI M., et al. : Effect of testosterone and estradiol in a man with aromatase deficiency. N. Engl.J. Med., 1997; 337 : 91-95. 
5. CARREAU S ., LEVALLET J. : L'aromatase testiculaire: du gène à la protéine. Andrologie, 1998; 8 (2) : 191-198.

6. CARREAU S., GENISSEL C., BILINSKA B., LEVALLET J. : The oestrogen sources in the testis and the reproductive tract of the male. Intern. J. Androl., 1999, 22: 211-223.

7. CARREAU S. : Estrogens and male reproduction . Folia Histochem.Cytobiol. , 2000a, 38 (2) : 47-52.

8. CARREAU S. , LEVALLET J. : Testicular estrogens and male reproduction. NIPS, $2000 \mathrm{~b}$, sous presse .

9. COUSE J.F., KORACH K.S. : Estrogen receptor null mice : what have we learned and where will they lead us? Endocr. Rev., 1999, 20 (3) : 358-417.

10. COUSE J.F;, CURTIS HEWITT S., BUNCH D.O. et al. : Postnatal sex reversal of the ovaries in mice lacking estrogen receptors a and $\mathbf{B}$. Science, 1999, 286 : 2328-2331.

11. DURKEE T.J., MUELLER M., ZINAMAN M. : Identification of estrogen receptor protein and messenger ribonucleic acid in human spermatozoa. Am. J. Obstet. Gynecol., 1998; 178 : 1288-1297.

12. EDDY E.M., WASHBURN T.F., BUNCH D.O. et al. : Targeted disruption of the estrogen receptor gene in male mice causes alteration of spermatogenesis and infertility. Endocrinology, 1996, 137 : 4796-4805.

13. ENMARK E., PELTO-HUIKKO M., GRANDIEN K. et al. : Human estrogen receptor- $B$ gene structure, chromosomal localization, and expression pattern. J.Clin.Endocrinol.Metab., 1997; $82: 4258-4265$.

14. ENMARK E., GUSTAFSSON J.A. : Oestrogen receptors-an overview. J. Inter. Med., 1999, 246 : 133-138.

15. FAUSTINI-FUSTINI M., ROCHIRA V., CARANI C. : Oestrogen deficiency in men: where are we today? Eur. J. Endocrinol., 1999, 140 : 111-129.

16. FISHER C.R., GRAVES K.? PARLOW A.F., SIMPSON E.R. : Characterization of mice deficient in aromatase (ArKO) because of targeted disruption of the cyp19 gene. Proc.Natl.Acad.Sci.USA, 1998, 95 : 6965-6970.

17. FISHER J.S., TURNER K.J., FRASER H.M., SAUNDERS P.T.K., BROWN D., SHARPE R.M. : Immunoexpression of aquaporin-1 in the efferent ducts of the rat and marmoset monkey during development, its modulation by estrogens, and its possible role in fluid resorption. Endocrinology, 1998, 139 : 3935-3945.

18. FOWLER K.A., GILL K., KIRMA N., DILLEHAY D.L., TEKMAL R.R. : Overexpression of aromatase leads to development of testicular Leydig cell tumors. Am. J. Pathol., 2000, 156 : 347-353.

19. GUNASEGARAM R., CHEW P.C.T., LOGANATH A., PEH K.L., RATNAM S.S. : A $\Delta 4$-3-keto pathway for testosterone synthesis in the human spermatozoa. Arch. Androl., 1998, $40: 49-57$.
20. GRUMBACH M.M., AUCHUS R.J. : Estrogen : consequences and implications of human mutations in synthesis and action. J. Clin. Endocrinol. Metab., 1999, 84 : 4677-4694.

21. GUSTAFSSON J.A. : Estrogen receptor ß- a new dimension in estrogen mechanism of action. J. Endocrinol., 1999, $163: 379-383$.

22. HARADA N. : Aromatase and intracinology of estrogen in hormone-dependent tumors. Oncology, 1999, $57: 7-16$.

23. HESS R.A., BUNICK D., LEE K H. et al. : A role for estrogens in the male reproductive sytem. Nature, 1997, $390: 509-512$.

24. HESS R.A., BUNICK D., LUBAHN D.B., ZHOU Q., BOUMA J. : Morphologic changes in efferent ductules and epididymis in estrogen receptor- $\alpha$ knockout mice. J. Androl., 2000, 21 : 107-121.

25. INKSTER S., YUE W., BRODIE A. : Human testicular aromatase: immunocytochemical and biochemical studies. J. Clin . Endocrinol . Metab., 1995, 80: 1941-1947.

26. JANULIS L., HESS R.A., BUNICK D. et al. : Mouse epididymal sperm contains active $\mathrm{P} 450$ aromatase which decreases as sperm traverse the epididymis. J. Androl., 1996, $17:$ 111-116.

27. JANULIS L., BAHR J.M., HESS R.A., JANSSEN S., OSAWA Y., BUNICK D.: Rat testicular germ cells and epididymal sperm contain active $\mathrm{P} 450$ aromatase. J. Androl., 1998, $19: 65-71$.

28. KATZ D., NIEDERBERGER C., SLAUGHTER FG.R.? COOINEY A.J. : Characterization of germ cell specific expression of the orphan nuclear receptor, germ cell nuclear factor. Endocrinology, 1997, $138: 4364-4372$.

29. LEVALLET J., BILINSKA B., MITTRE H., GENISSEL C., FRESNEL J., CARREAU S. : Expression and immunolocalization of functional cytochrome P450 aromatase in mature rat testicular cells. Biol. Reprod., 1998, 58 : 919-926.

30. LUCONI M., MURATORI M., FORTI G., BALDI E.: Identification and characterization of a novel functional estrogen receptor on human sperm membrane that interferes with progesterone effects. J. Clin. Endocrinol. Metab., 1999, 84 (5) : 1670-1678

31. MAHATO D., GOULDING E.H., KORACH K.S., EDDY E.M.: Spermatogenic cells do not require estrogen receptor $\alpha$ for development or function. Endocrinology, 2000, $141: 1273-1276$.

32. MORISHIMA A., GRUMBACH M.M., SIMPSON E.R., FISCHER C., QIN K. Aromatase deficiency in male and female siblings caused by a novel mutation and the physiological role of estrogens. J. Clin. Endocrinol. Metab., 1995, 80 : 3689-3698.

33. MOSSELMAN S., POLMAN J., DIJKEMA R. ERß: identification and characterization of a novel human estrogen receptor. FEBS Lett., 1996, 392 : 49-53. 
34. PAYNE A.H., KELCH R.P., MUSICH S.S., HALPERN M.E. : Intratesticular site of aromatization in the human. J.Clin.Endocrinol. Metab., 1976, 42 : 1081-1087.

35. RAVNIK S.E., WOLEGEMUTH D.J. : Regulation of meiosis during mammalian spermatogenesis : the A-type cyclins and their assiociated cyclin-dependent kinases are differentially expressed in the germ cell lineage. Dev. Biol., 1999, 207 : 408-418.

36. REVELLI A., MASSOBRIO M., TESARTIK J. : Nongenomic actions of steroid hormones in reproductive tissues. Endocr. Rev., 1998, 19 : 3-17.

37. ROBERTSON K.M., O'DONNELL L., JONES M.E.E., et al. : Impairment of spermatogeneis in mice lacking a functional aromatase (CYP19) gene. Proc Natl Acad Sci USA, 1999, 96 : 7986-7991.

38. ROBERTSON K.M., O'DONNELL L., SIMPSON E.R., JONES M.E.E. : The late onset phenotype of the male aromatase knockout (ArKO) mouse. XI th European Workshop on Molecular and Cellular Endocrinology of the Testis. Saint-Malo 13-17 Mai 2000, G11.

39. SAEZ J.M. : Leydig cells: endocrine, paracrine and autocrine regulation. Endocr.Rev., 1994, 15 : 574-626.

40. SAUNDERS P.T.K., SCOBIE G., MILLAR M.R., MacPHERSON S., EVANS L.W., GROOME N.P. : Differential expressio of oestrogen receptor beta types 1 and 2 in human testis.XI th European Workshop on Molecular and Cellular Endocrinology of the Testis. Saint-Malo 13-17 Mai 2000, G18.

41. SHARPE RM. Do males rely on female hormones. Nature, 1997, 390: 447-448

42. SHETTY G., KRISHNAMURTHY H., KRISHNAMURTHY H.N., BHATNAGAR S., MOUDGAL R.N. : Effect of estrogen deprivation on the reproductive physiology of male and female primates. J. Steroid Biochem. Molec. Biol. 1997, 61 (3-6) : 157166.

43. SHETTY G., KRISHNAMURTHY H., KRISHNAMURTHY H.N. et al. : Effect of long term treatment with aromatase inhibitor on testicular function of adult male bonnet monkeys. Steroids, 1998, 63 : 414-420.

44. SHOZU M., ZHAO Y., BULUN S.E., SIMPSON E.R.: Multiple splicing events involved in regulation of human aromatase expresion by a novel promoter I.6. Endocrinology, 1998, 139 : 1610-1617.

45. SHUGHRUE P.J., LANE M.V., SCRIMO P.J., MERCHENTHALER I. : Comparative distribution of etrogen receptor- $\alpha(\mathrm{ER}-\alpha)$ and $\beta(\mathrm{ER}-\beta) \mathrm{mRNA}$ in the rat pituitary, gonad, and reproductive tract. Steroids, 1998, $63: 498-504$.

46. SIMPSON E.R., MAHENDROO M.S., MEANS G.D. et al. : Aromatase cytochrome P450, the enzyme responsible for estrogen biosynthesis. Endocr.Rev., 1994, $15: 342-355$.
47. SIMPSON E.R., MICHAEL M.D., AGARWAL V.R., HINSHELWOOD M.M., BULUN S.E., ZHAO Y . : Expression of the CYP 19 (aromatase) gene: an unusual case of alternative promoter usage. FASEB J., 1997, $11: 29-36$.

48. SIMPSON E.R., RUBIN G., CLYNE C. et al. : Local estrogen biosynthesis in males and females. Endocr. Related Cancer, 1999, 6 : 131-137.

49. SMITH EP, BOYD J, FRANK GR, et al. Oestrogen resistance caused by a mutation in the oestrogen receptor gene in man. New Engl. J. Med., 1994; 331: 1056-1061.

50. TURNER K.J., MORLEY M., ATANASSOVA N., SWANSTON I.D., SHARPE R.M. : Effect of chronic administration of an aromatase inhibitor to adult male rats on pituitary and testicular function and fertility. J. Endocrinol., 2000, 164 : 225-238.

51. van PELT A.M.M., de ROOIJ D.G., van der BURG B., van der SAAG P.T., GUSTAFSSON J.A., KUIPER G.G.J.M. : Ontogeny of estrogen receptor- $\beta$ expression in rat testis. Endocrinology, 1999, 140:478483 .

\author{
ABSTRACT \\ Estrogens and male reproduction \\ S.CARREAU
}

Aromatase is the terminal enzyme responsible for estrogen biosynthesis. Besides somatic cells, the aromatase gene expression and its transduction in a fully active protein in germ cells of rodent testes in one hand, the widespread distribution of estrogen receptors (ER $\alpha$ and ERß) in the genital tract of the male in an other hand, are clearly in favour of a physiological role for estrogens in the regulation of mammalian testicular functions. In mouse and man, the aromatase deficiency is associated with severe bone maturation problems and sterility. Therefore, together with gonadotrophins and androgens, estrogens (or the balance androgens / estrogens) likely play a physiological role (either directly or via testicular somatic cells) in maintenance of male gonadal functions and obviously, several steps are concerned especially the spermatid production (both in terms of quality and number) and epididymal sperm maturation.

Key words : Estrogens, spermatogenesis, estrogen receptors, aromatase, fertility, man 